\title{
1 Experiments and simulations on short chain fatty acid production in a colonic
}

\section{2 bacterial community}

3

16 \#Address correspondence to: Daniel Segrè, dsegre@,bu.edu and Todd Thorsen,

17 thorsen@11.mit.edu

18

B.Y. and I.D. contributed equally to this work.

DISTRIBUTION STATEMENT A. Approved for public release. Distribution is unlimited.

This material is based upon work supported by the Assistant Secretary of Defense for Research and Engineering under Air Force Contract No. FA8702-15-D-0001. Any opinions, findings, conclusions or recommendations expressed in this material are those of the author(s) and do not necessarily reflect the views of the Assistant Secretary of Defense for Research and Engineering. 


\section{Abstract}

22 Understanding how production of specific metabolites by gut microbes is modulated by

23 interactions with surrounding species and by environmental nutrient availability is an important

24 open challenge in microbiome research. As part of this endeavor, this work explores interactions

25 between $F$. prausnitzii, a major butyrate producer, and B. thetaiotaomicron, an acetate producer,

26 under three different in vitro media conditions in monoculture and coculture. In silico Genome-

27 scale dynamic flux balance analysis (dFBA) models of metabolism in the system using

28 COMETS (Computation of Microbial Ecosystems in Time and Space) are also tested for

29 explanatory, predictive and inferential power. Experimental findings indicate enhancement of

30 butyrate production in coculture relative to $F$. prausnitzii monoculture but defy a simple model

31 of monotonic increases in butyrate production as a function of acetate availability in the medium.

32 Simulations recapitulate biomass production curves for monocultures and accurately predict the

33 growth curve of coculture total biomass, using parameters learned from monocultures,

34 suggesting that the model captures some aspects of how the two bacteria interact. However, a

35 comparison of data and simulations for environmental acetate and butyrate changes suggest that

36 the organisms adopt one of many possible metabolic strategies equivalent in terms of growth

37 efficiency. Furthermore, the model seems not to capture subsequent shifts in metabolic activities

38 observed experimentally under low-nutrient regimes. Some discrepancies can be explained by

39 the multiplicity of possible fermentative states for $F$. prausnitzii. In general, these results

40 provide valuable guidelines for design of future experiments aimed at better determining the

41 mechanisms leading to enhanced butyrate in this ecosystem. 


\section{Importance}

45 Studies associating butyrate levels with human colonic health have inspired research on

46 therapeutic microbiota consortia that would optimize butyrate production if implanted in the

47 human colon. Faecalibacterium prausnitzii is commonly observed in human fecal samples and

48 produces butyrate as a product of fermentation. Previous studies indicate that Bacteroides

49 thetaiotaomicron, also commonly found in human fecal samples, may enhance butyrate

50 production in F. prausnitzi when the two species are co-localized. This possibility is

51 investigated here under different environmental conditions using experimental methods paired

52 with computer simulations of the whole metabolism of bacterial cells. Initial findings indicate

53 that interactions between these two species result in enhanced butyrate production. However,

54 results also paint a nuanced picture, suggesting the existence of a multiplicity of equivalently

55 efficient metabolic strategies and complex interactions between acetate and butyrate production

56 in these species that appear highly dependent on specific environmental conditions.

\section{Introduction}

It is increasingly recognized that metabolites produced by the resident microbiota of the

60 colon have a major influence on host physiology (1). Dietary substrates dramatically influence

61 the amount and type of these metabolites produced (2). For instance, fermentation of

62 carbohydrates produces a number of bioactive compounds, most notably short chain fatty acids

63 (SCFA) such as butyrate, that have been demonstrated to shape the gut microenvironment, serve

64 as an energy source for the colonic epithelium, and influence disease through anti-inflammatory,

65 lipogenic, and anti-apoptotic effects (3-6). 
The production of metabolites in a microbial community has been suggested to be heavily modulated by interactions among its members. These interactions manifest in a variety of modes, ranging from competitive or predatory to commensal and mutualistic exchanges (7). Additionally, many microbes in nature exist in spatially defined structures (8), such as the mucosal layer of the gut. Spatial assortment of cells creates locally heterogeneous subpopulations with varying access to resources that can also modulate inter- and intracommunity behavior (9). A major goal of ongoing efforts in human microbiome research (10) is to gain enough predictive and quantitative understanding of inter-microbial interactions (11) and

74 of the metabolic interplay between microbiota and host (12) to be able to understand the effects

75 of the microbiome on human health. These capabilities could greatly facilitate successful design

76 of therapeutic strategies for microbiome-related diseases.

to improve human health are, however, limited by insufficient understanding of the nature of the mechanisms underlying microbial interactions and the way these interactions affect microbiome dynamics. Anaerobic in vitro, in vivo and ex vivo experiments capable of probing systems similar

81 to the human colonic environment are difficult and expensive. Previous work uncovering

82 fundamental properties of SCFA-producing bacteria and their symbiotic partners has used

83 Faecalibacterium prausnitzii as a model system (13-21). This is motivated by the high

84 prevalence of $F$. prausnitzii as a commensal bacterium in the human large intestine (22) and the

85 role it plays as one of the major butyrate producers (23). Among the bacteria used for coculture

86 studies, a common gut commensal, Bacteroides thetaiotaomicron, has been chosen in both

87 experimental (24) and computational (25) analyses of SCFA production. In particular,

88 experimental efforts to grow $F$. prausnitzii in coculture with $B$. thetaiotaomicron have suggested 
89 enhancement of butyrate production in coculture relative to $F$. prausnitzii monoculture (24).

90 However, the data in this study was obtained only for a single time point and no information on

91 the dynamics of the biomass or butyrate production was provided. In general, to our knowledge,

92 no comparison has been previously made between experimentally measured time-courses of the

93 biomass of these species and their respective metabolic dynamics, when grown individually and

94 in co-culture.

In parallel, computational work based on metabolic network analyses has led to the

96 construction of genome scale models for each of these bacterial species, and to a computational

97 assessment of their metabolic capabilities (26-28). The modeling approach used in these studies,

98 often referred to as constraint-based modeling (or stoichiometric modeling) is based on

99 simplifying assumptions about the intracellular dynamics of metabolism. It enables quantitative

100 predictions of the intracellular and exchange fluxes, in addition to the growth rate of different

101 species. In particular, flux balance analysis (FBA) (29), can be used to calculate the flow of

102 metabolites through a metabolic network, making it possible to predict the growth rate of an

103 organism or the rate of production of important metabolites (30) ( see also (31) for a

104 comprehensive review of different approaches). While the reconstructed networks and the

105 modeling tools used for making these predictions vary widely in accuracy and predictive power,

106 the formal representation of metabolism into these mathematical structures and codification of

107 multi-level processes into algorithms have sparked a revolution in systems biology of

108 metabolism, enabling precise hypothesis testing, and the formulation of genome-scale based

109 community modeling. In the context of human gut microbiome studies and inter-species

110 interactions, modeling work has been shown in particular to provide insight into the stability of

111 biofilm forming communities (25). 
In order to make comparisons between computational predictions and experimental

113 time-course data, it is important to be able to connect detailed knowledge of the intracellular

114 metabolism of individual organisms to the dynamic metabolic changes occurring in the

115 surrounding environment. An extension of FBA capable of these types of calculations is

116 dynamic FBA (or dFBA)(32). Harcombe et al. (33) developed a computational framework

117 specifically designed to help predict the spatio-temporal behavior of synthetic microbial

118 consortia. This system, known as Computation of Microbial Ecosystems in Time and Space

119 (COMETS) (33), generates predictions of biomass growth curves as well as detailed time

120 dynamics of the concentrations of all nutrients and metabolites in the environment. COMETS

121 has been shown to accurately predict the behavior of small artificial ecosystems.

Despite the availability of these experimental data and computational tools, many

123 fundamental features of the interactions between $F$.prausnitzii and B. thetaiotaomicron, as well

124 as our capacity to predict clinically relevant variables, remain unexplored. While previous

125 studies have computationally and experimentally analyzed the metabolic capabilities of each of

126 these bacteria individually $(26,27)$ and the dependence of these and other bacteria upon different

127 oxygen levels (25), no direct comparison of experimental and computational time course data for

128 this consortium under varying conditions has been presented before. In particular, no attempt

129 has been made to recapitulate or predict these time-courses with dynamic computational models.

Here, we provide novel insight into the $F$. prausnitzii - B. thetaiotaomicron model

132 system by combining new experimental measurements of bacterial biomass and environmental

133 metabolites with COMETS-based computer simulations. We performed a series of anaerobic in

134 vitro experiments involving monocultures and cocultures of $F$. prausnitzii and $B$.

135 thetaiotaomicron grown in three different media, and found increased butyrate production in co- 
136 culture relative to monoculture under high glucose and acetate concentrations. Upon fitting of six

137 parameters for metabolic uptake kinetics in monocolture, COMETS simulations were able to

138 recapitulate biomass time courses in monoculture and predict combined biomass time courses for

139 coculture. Model predictions for butyrate, however, portray a more complex picture. Accurate

140 predictions of initial butyrate production rate do not hold at longer times due to the existence of

141 multiple alternative optima in the flux states and the history-dependence of the dynamical

142 predictions. Strong sensitivity of the butyrate production curves to specific concentrations of

143 nutrients, including phosphate, provide insight into the complexity of these metabolic exchanges,

144 and valuable guidance for future experimental and modeling work.

146 Results

147 In vitro and in silico coculture biomass dynamics under different nutrient limitations

148 We initially characterized anaerobic growth of B. thetaiotaomicron and F. prausnitzii

149 individually and in coculture, under different levels of carbon availability (low, medium, high,

150 see Methods). In addition to glucose, acetate was added proportionally, mimicking the

151 fermentative activity of the rest of the microbiota (13). The presence of acetate in the medium

152 also allowed us to assess how, even in the absence of $B$. thetaiotaomicron, $F$. prausnitzii

153 responds to varying acetate availability.

Monoculture growth for $B$. thetaiotaomicron appears sensitive to the amount of carbon

155 provided (Fig. 1). Growth rate and yield increase in medium acetate/glucose medium compared

156 to low acetate/glucose concentrations. No clear increase in biomass occurred when carbon

157 abundance was increased from medium to high levels. The amount of $F$. prausnitzii biomass in

158 monoculture appears insensitive to increase in initial acetate/glucose levels. The combined 
159 biomass growth curves of the coculture (measured as a collective OD) closely tracks OD curves

160 of $B$. thetaiotaomicron, suggesting a prominent role of this bacterium in the consortium. This

161 observation is consistent with previous experiments (3) in which the combined coculture biomass

162 OD of $B$. adolescentis and $F$. prausnitzii mirrors the OD of the $B$. adolescentis monoculture,

163 suggesting that some features of that consortium may be similar to the one studied here, even

164 across different spatial scales and environmental settings.

In parallel to the experimental measurements, we implemented in silico simulations of

166 the same monocultures and cocultures, using previously published genome-scale metabolic

167 models for the two bacteria $(26,27)$. In particular, we used COMETS (33) to test whether (i)

168 parameters from the literature, combined with minimal fitting of unknown parameters, would

169 recapitulate the observed monoculture behavior and (ii) models tuned for monoculture

170 experiments would be adequate to predict the outcome of the coculture experiment.

171 Superimposed on the experimental data, Fig. 1 shows the biomass dynamics as

172 simulated in COMETS. The monoculture simulations were supplemented with empirical

173 knowledge of uptake $K_{M}$ and fitting of $V_{\max }$ values. After selecting initial kinetic parameter

174 values based on previously determined corresponding parameters for the phosphotransferase

175 (PTS) transporter $(21,34)$, a sensitivity analysis allowed us to identify $V_{\max }$ values that provide

176 best fit of growth curves to monoculture (Fig. 2). This calibration step, similar to that previously

177 performed in (35), produces simulated OD curves that broadly agree with the experimentally

178 measured points (see root-mean-squared error (RMSE) in Table 1). Using these parameters for

179 COMETS simulations of cocultures at the experimentally estimated initial biomass abundances,

180 coculture predictions track coculture experimental data closely, as shown by predictive RMSE

181 values (Table 1). 


\section{Coculture conditions impact the average rate of butyrate production in experiments} measured the extracellular aboundance of butyrate and acetate. In Fig. 3, the average amount of

187 and high initial acetate/glucose concentrations, but not in the low concentration conditions. In

188 low acetate/glucose conditions the butyrate production rate in coculture appears suppressed

189 relative to the one in monoculture. Box's approach in (36), applying ANOVA to summary

190 statistics describing growth curves, was used to quantify the statistical significance of the

191 difference in butyrate production curves across different treatments, i.e.: (i) monoculture vs.

192 coculture conditions (mono/co) and (ii) the three different initial acetate/glucose initial

193 concentrations (initial glu/ac). Tables 2 and 3 show that the average rate of butyrate production

194 in F. prausnitzii is significantly altered in mono vs. coculture conditions but not across the

195 different initial abundances of acetate and glucose. The initial acetate/glucose concentrations, but

196 not mono vs. coculture conditions, significantly change the average rate of acetate production

197 from B. thetaiotaomicron.

Interpretation of the above assessments of butyrate and acetate production is limited by

199 the lack of experimental knowledge of the precise amount of biomass of each species in the

200 coculture experiments. In particular, in absence of further laborious organism-specific data, it is

201 impossible to determine whether significant changes in the level of the butyrate curves are due to

202 F. prausnitzii producing more butyrate at the cellular level in the coculture, or whether the

203 increase is due to an increase in F. prausnitzii total biomass, enabled by coculture conditions, or

204 both. Under these circumstances, COMETS-predicted biomass estimates for each species from 
coculture simulations (Fig. 6B) enabled hybrid computational-experimental estimates of biomass-normalized butyrate (Fig. 6A) and acetate (Fig. S2) production curves.

208 for the unnormalized curves, with several exceptions. The average rate of both SCFAs are

209 significantly affected by mono vs. coculture conditions in the normalized curves. These results

210 imply that $B$. thetaiotaomicron stimulates butyrate production in $F$. prausnitzii on a per cell basis

211 rather than by stimulating $F$. prausnitzii biomass production. B. thetaiotaomicron biomass-

212 normalized acetate production curves are not significantly changed in average rate by initial

213 glucose/acetate levels, in contrast to the non-normalized curves. As a note of caution, it is

214 important to stress that the normalization relative to untested predicted abundances of individual

215 species in co-culture should be considered putative. At the same time, it could be viewed as a

216 valuable strategy for integrating experimental and computational data towards the formulation of

217 new hypotheses.

219 The multiplicity of fermentation states with optimal efficiency influences SCFA time-course 220 predictability

Figure 3 shows that COMETS accurately recapitulates the early stages of

222 accumulation of extracellular butyrate. After 5 hours of growth, however, the picture becomes

223 more complex. Simulation results at these times suggest that the regulation of butyrate and

224 lactate pathways may play a major role in the final outcome of the secreted butyrate. To

225 understand these results, the butyrate fermentation process in F. prausnitzii was revisited with

226 additional computational analyses, specifically focused on alternative fermentation pathways.

227 Three competing fermentation pathways exist in the curated metabolic network of F. prausnitzii.

228 Pyruvate is fermented to one of the three products (27): (i) lactate, by D-lactate dehydrogenase 
229 (reaction ID: $\mathrm{LDH}_{\mathrm{D}}$ ), (ii) formate, by pyruvate-formate lyase (reaction ID: PFL) or (iii) butyrate,

230 by butyryl-CoA:acetate CoA-transferase (reaction ID: BTCOAACCOAT). Butyryl-CoA in turn

231 is produced from acetyl-CoA (reaction ID: BTCOADH) and acetyl-CoA is converted from

232 pyruvate by pyruvate:ferredoxin oxidoreductase (reaction ID: POR4i). The balance of metabolic

233 flow through these three closely coupled fermentation pathways can significantly impact the

234 production of butyrate in $F$. prausnitzii, depending on environmental conditions.

As shown in Figs. 3 and S4, and described in the methods section, COMETS, in its

standard formulation, switches among these competing pathways. In particular, Figure S3 shows

237 that $F$. prausnitzii switches from predominantly butyrate production activity at time zero to

238 lactate secretion after 5 hours. This switch coincides with the transition from a single solution

239 point of the FBA optimization at the early stages of the growth, to conditions where the FBA

240 optimization algorithm has the freedom of choosing between a multitude of flux solution points,

241 all corresponding to the same biomass growth rate. This multiplicity of equivalently efficient

242 steady states (multiple alternative optima, also described in (37)) is best highlighted by

243 systematically imposing, at each time point, additional features in the dFBA solution process. In

244 particular, in analogy with flux variability analysis (38), we re-run the COMETS simulations by

245 adding a secondary objective function at each time point. After maximizing for growth, the

246 algorithm fixes the growth rate to the identified maximum, and subsequently searches for the

247 solution that maximizes or minimizes the secretion flux of one of the organic acids, such as

248 butyrate. Correspondingly, the butyrate secretion flux can be represented in the form of two

249 curves of butyrate concentration extremes (Fig. 3). Notably, in most of the cases, the

250 experimentally measured butyrate concentrations occupy a place between these two extremes.

251 Nutrient limitations seem to be a strong determinant of this multiplicity of alternative optima. 
252 The system is particularly sensitive to phosphate concentration, as shown in Figs. 4 and S4, and

253 is probably due to the strong coupling to phosphate in all butyrate producing pathways. These

254 simulation results therefore suggest that regulation of the fermentation pathways in $F$. prausnitzii

255 influence butyrate production under different environmental conditions.

256 The simulated acetate production shown in Fig. 5 tracks experimental time courses for

257 both $B$. thetaiotaomicron and F. prausnitzii monoculture, particularly in the medium and high

258 inital acetate/glucose conditions. Simulated time courses fall within error bars for average

259 experimental observations for all time points on the curves. The early stages of the coculture

260 simulations also closely track experiments. After 5 simulated hours, however, opposite trends in

261 acetate concentrations appear to mirror the discrepancies with experiment in the butyrate

262 simulations. While the experiments show depletion, the simulations result in buildup of acetate

263 in the late stages of the simulations. This inconsistency is also potentially explainable by a

264 metabolic switch in F. prausnitzii, resulting in less acetate consumption to produce butyrate.

266 Discussion

We analyzed experimentally and computationally the possible effects of a symbiotic

268 partner (B. thetaiotaomicron) and of environmental conditions (amount of glucose and acetate)

269 on the biomass of the gut bacterium F.prausnitzii, as well as its capacity to produce butyrate. In

270 monoculture, $F$. prausnitzii seems to continue producing butyrate even after the cells reach

271 stationary phase (at around 10 hours), suggesting that maintenance processes keep fueling the

272 butyrate production pathways. Another feature of the monoculture is the relatively low

273 sensitivity of biomass and butyrate production to glucose and acetate concentrations. In contrast,

274 biomass and butyrate seem to more strongly depend on the environment in the presence of $B$. 
275 thetaiotaomicron in the coculture experiments (Figure 3), although this difference is not 276 statistically significant, based on ANOVA.

277 The possibility of metabolic cross-feeding between $F$. prausnitzii and $B$.

278 thetaiotaomicron has been suggested in previous studies (24). In some of these studies, acetate

279 production by $B$. thetaiotaomicron is proposed to mediate the interaction between the two

280 bacteria, facilitating an increased butyrate production by $F$. prausnitzii. While it is likely that

281 indeed acetate plays a key role in the interaction between the two bacteria, the results of our

282 study suggest a more complex mode of interaction: First, the statistically significant increase in

283 butyrate production we observe in coculture does not seem to increase monotonically with the

284 amount of glucose and acetate, suggesting that either the two carbon sources are saturated (24),

285 or that acetate exchange is not the only factor dominating butyrate production. Second, as

286 demonstrated by our COMETS dFBA simulations, the stoichiometry of $F$. prausnitzii suggests

287 that multiple alternative growth optima are possible (depending on whether or not other nutrients

288 - most notably phosphate - are limiting in the medium). These different optima can differ

289 substantially in their combination of fermentation products, thus making the stoichiometry-based

290 prediction of a specific rate of butyrate production impossible. Instead, only a range of

291 production rates can be predicted at any given time. This hypothesized degree of freedom in

292 fermentative pathways could in principle be used by $F$. prausnitzii to modulate its metabolic

293 activity and its butyrate production rate in response to external signals. Future studies using

294 dFBA for studying $F$. prausnitzii could use additional constraints (e.g. total flux capacity (38) or 
295 regulatory information (38)) to refine the predictions, and to systematically test the effect of

296 different nutrient limitations and growth media on butyrate production.

298 of the two species in coculture, in the absence of experimental observations. Although the

299 accuracy of the COMETS relative biomass estimates were not confirmed using experimental

300 data, its ability to predict the total biomass in coculture using parameters learned from

301 monoculture conditions lends credence to these estimates. Follow-up experiments to fully vet

302 model accuracy in determining relative biomass in consortia would be invaluable towards

303 building confidence in hybrid computational-experimental approaches like the one demonstrated

304 here.

Methods and Materials

307 COMETS Simulation Configuration The metabolic network models we use in this work for

308 Bacteroides thetaiotaomicron strain VPI-5482 and Faecalibacterium prausnitzii strain A2-165

309 were published and made publicly available by Heinken et al. in (26) and (27). The COMETS

310 simulation framework is implemented in Java and described in (33). R and Matlab scripts

311 transform COMETS outputs to time-course plots. The 3D volume in these simulations contains 5

$312 \mathrm{~mL}$ of isotropic medium and biomass. COMETS' ability to model spatial differences in

313 microbial systems is not explored in these preliminary simulations. Similarly, the dFBA settings,

314 other than the ones mentioned below, were set at their default values as implemented in

315 COMETS. The FBA parsimonious optimization was performed using the GUROBI optimizer,

316 with a primary maximization of the biomass growth rate and a secondary minimization of the

317 absolute values sum of the metabolic fluxes. The butyrate secretion analysis also included 
318 additional maximization and/or minimization of butyrate, lactate and formate uptake. Simulation

319 run time was 24 hours, with each time step set to 0.01 hour. The death rate was set to zero.

320 The uptake of nutrients was modeled as a saturation Michaelis-Menten curve with two

321 adjustable parameters, maximum uptake flux, $\mathrm{V}_{\max }$, and the Michaelis constant $\mathrm{K}_{\mathrm{M}}$. Our choice

322 of parameters for the uptake curve was guided by values provided in the original publications of

323 the models and as reported in the literature $(39,40)$. The glucose uptake in $F$. prausnitzii, for

324 example, is governed by PTS transporter (27) with reported $\mathrm{K}_{\mathrm{M}}$ values up to $8.7 \mathrm{mM}$ (34). These

325 starting values for the uptake parameters where additionally fine-tuned by fitting the single-

326 species simulations results for the OD to the corresponding experimental curves. Error!

327 Reference source not found. shows the fitting procedure for $B$. thetaiotaomicron and $F$.

328 prausnitzii respectively. In the case of $B$. thetaiotaomicron a single value for the maximum

329 uptake parameter was sufficient to fit the growth curves. The accepted value minimized the

330 composite reduced chi-squared for all three growth conditions. In the case of $F$. prausnitzii, we

331 used two values for the maximum uptake. Starting with the value for $\mathrm{K}_{M}$ we fitted the glucose

332 and acetate uptake, and then performed a fine tuning for the rest of the metabolites/nutrients.

333 Parameter values are shown in Table 6.

334 Metabolic activity in the $F$. prausnitzii model showed a nutrient concentration

335 dependent shift, most sensitive to phosphate depletion, from butyrate producing pathway, to a

336 lactate producing one, shown in Fig. S3. This shift is characterized by a single solution point of

337 the FBA optimization sequence, both for minimized and maximized butyrate secretion, at high

338 values of phosphate concentration, shown in Fig. S4, corresponding to the initial time in the

339 dFBA simulation. As the substrate is depleted of the nutrients, the system obtains multiple

340 optimal solution points, with the difference in the butyrate production depending on the 
341 secondary optimization of butyrate secretion (Fig. S4) providing a range of possible butyrate

342 secretion rates at the later stages of the dFBA simulations. The complete set of input as well as

343 simulations output files can be found in the supplement. COMETS is available to download at

344 comets.bu.edu.

346 ANOVA Methodology Box's method (36) for describing and quantifying differences in growth

347 curves was implemented as follows. The average rate/level statistic is computed as the average

348 of the measurements from the first time point concentration measurement subtracted from the

349 average of the measurements from the last divided by the total time. The rate of butyrate

350 production for each time bin was computed similarly and the average rate was subtracted from

351 these for the set of rate deviations that define the shape statistic.

We use the Bonferonni corrected significance level of 0.0042 in this study with twelve

353 comparisons (

354 single comparisons. The small number of replicates in our study (3 replicates), results in

355 relatively low power for ANOVA tests. Insignificant results in our ANOVA analysis may

356 therefore derive from low power, randomness or some combination of the two (41) for both the

357 shape and level ANOVA results. Additionally, violations of homogeneous measurement

358 covariance matrices and/or normally distributed prediction errors with zero mean could also

359 result in pessimistically biased significance estimates. In particular, violation of homogeneous

360 covariance matrices may negatively bias shape ANOVA results (42). ANOVA results for level

361 have been shown to be robust to violations of this assumption (43).

362 We did not test our data for violations of these two conditions because tests for normality

363 and equal variance are themselves inconclusive with small sample sizes. Given that violations of 
364 homogeneous measurement covariance matrices can negatively bias significance results in shape

365 ANOVA, the insignificant results in this study should be probed further with larger sample sizes.

366 Because level ANOVA results have been shown to be robust to violations of homogeneous

367 measurement covariance matrices (43), we are confident in the finding of significant differences

368 between monoculture and coculture metabolite production curves in level/average rate. As is

369 always the case, however, follow-up studies with larger sample sizes would be advised to both

370 test reproducibility and lend more power to ANOVA results.

372 In Vitro Experimental Configuration/OD600 Analysis Bacteria were cultured in Yeast

373 Casitone (YC) medium, with three different concentrations of supplemented acetic acid/glucose.

374 "Low" condition: 5.551mM acetic acid, .1\% glucose. "Medium" condition: $27.754 \mathrm{mM}$ acetic

375 acid, .5\% glucose. "High" condition: 55.507mM acetic acid, 1\% glucose. All media were

376 adjusted to $\mathrm{pH} 6.8$ before autoclaving.

377 Bacterial cultures were started in anaerobic conditions from glycerol stocks stored at -

$37880^{\circ} \mathrm{C}$ in $3 \mathrm{~mL}$ of "Medium" YC medium. After overnight culture, OD600 was measured, and the

379 cultures were diluted to the nominal OD starting points in $5 \mathrm{~mL}$ of the three different $\mathrm{YC}$

380 formulations.

381 The following cultures were started with the initial OD600 values as noted:

382 OD600 .02 B. thetaiotamicron monoculture

383 OD600 .08 F. prausnitzii monoculture

384 OD600 .02 B. thetaiotamicron AND OD600 .08 F. prausnitzii coculture

A baseline $200 \mu \mathrm{L}$ aliquot was taken from each culture, measured by OD600, and stored

386 at -80 for later MS analysis. Subsequent $200 \mu \mathrm{L}$ aliquots were collected and measured by OD600 
at 2, 4, 6, 8, 10 and 24 hours and stored for later analysis. The above procedure was repeated in triplicate, yielding three observations per time point.

MSMS Analysis A flow injection analysis electrospray ionization mass spectrometry (FIA ESI

391 MSMS) method was used for quantitative detection of short chain fatty acids (SCFA). Acetic,

392 propionic, butyric and succinic acid were derivatized with 3-nitrophenylhydrazine in the

393 presence of $\mathrm{N}$-(3-dimethylaminopropyl)-N'-ethylcarbodiimide and pyridine and detected by a

394 mass spectrometer as a 3-nitrophenylhydrozones in MRM (multiple reaction monitoring) MSMS

395 mode, as described by J. Han et al. (44). To increase precision and robustness of the method, 3-

396 methylbutyric-2-2-d2 acid, acetic acid-2-13C and propionic acid -1-13C were used as an

397 internal standards. Quantitation was done by external standards calibration, where instrument

398 response for the analyte was measured as a ratio between analyte's and internal standard's peak

399 areas. The FIA technique did not utilize LC column but rather a direct injection of the sample

400 into an ESI probe of the mass spectrometer and this decreased time of analysis per sample to two

401 minutes. The FIA ESI MSMS for the detection of SCFA is sensitive with the limit of detection

402 for acetic, propionic, butyric and succinic acids at 4, 3, 0.6 and $1.4 \mu \mathrm{M}$ respectively. The

403 accuracy of the method was between $98-102 \%$.

Reagents LC MS grade acetonitrile and water were purchased from VWR (Radnor, PA, USA).

406 N-(3-dimethylaminopropyl)-N'-ethylcarbodiimide $\mathrm{HCl}, 3$-nitrophenylhydrazine $\mathrm{HCl}$, pyridine,

407 acetic acid, propionic acid, butyric acid, succinic acid, acetic acid 13C, and propionic acid 13C

408 were purchased from Sigma-Aldrich (St Luis, MO, USA). 3-methylbutyric-2-2-d2 acid was

409 purchased from CDN Isotopes (Quebec, CN). 
411 FIA MS/MS system An Agilent infinity capillary LC pump with micro-autosampler and

412 thermostat (Agilent Technologies, Santa Clara, CA, USA) coupled to AB Sciex 4000 Q-TRAP

413 triple- quadrupole mass spectrometer ( AB Sciex, Concord, Ontario, CN) was used for the

414 analysis. The flow solvent - five percent water and ninety five percent acetonitrile was delivered

415 to a mass spectrometer ESI probe at the rate of $350 \mu \mathrm{L} / \mathrm{min}$. Samples for flow injection analysis

416 were derivatized on the Agilent polypropylene 96 well plate and injected into mass spectrometer

417 with injection volume of $40 \mu \mathrm{L}$. Following conditions for the AB Sciex Q-TRAP 4000 were used

418 for analysis: source temperature $400^{\circ} \mathrm{C}$, source gas $40 \mathrm{~L} / \mathrm{min}$, curtain gas $10 \mathrm{~L} / \mathrm{min}$, ESI capillary

419 voltage was set at -4500 volts. Data were acquired in negative polarity multiple reactions

420 monitoring (MRM) mode for the MRM transitions specified in the Table S1.

\section{Data Availability}

423 Data files and scripts used to generate the figures presented in this paper can be found in a zipped

424 directory (Yu_etal_Data_and_Scripts.zip) downloadable at https://github.com/segrelab/Fprau-

425 Btheta-2018. This directory contains the experimental data and script for statistical analysis and

426 for generating the figures (DATA_AND_FIGURE_SCRIPTS subdirectory), and COMETS input

427 and output files (SIMULATIONS_INPUTS_AND_OUTPUTS subditrectory). The in silico

428 experiments were generated using COMETS v.2.5.8, which is freely available at

429 http://comets.bu.edu. 
432 DS and ID acknowledge funding from the Defense Advanced Research Projects Agency

433 (Purchase Request No. HR0011515303, Contract No. HR0011-15-C-0091), the NIH

434 (5R01DE024468, R01GM121950 and Sub_P30DK036836_P\&F), and the Boston University

435 Interdisciplinary Biomedical Research Office. This material is based upon work supported by

436 the Assistant Secretary of Defense for Research and Engineering under Air Force Contract No.

437 FA8702-15-D-0001. Any opinions, findings, conclusions or recommendations expressed in this

438 material are those of the author(s) and do not necessarily reflect the views of the Assistant

439 Secretary of Defense for Research and Engineering.

440

\section{References}

442 1. Lozupone CA, Stombaugh JI, Gordon JI, Jansson JK, Knight R. 2012. Diversity, stability

443 and resilience of the human gut microbiota. Nature 489:220-230.

444 2. Yatsunenko T, Rey FE, Manary MJ, Trehan I, Dominguez-Bello MG, Contreras M,

445 Magris M, Hidalgo G, Baldassano RN, Anokhin AP, Heath AC, Warner B, Reeder J,

446 Kuczynski J, Caporaso JG, Lozupone CA, Lauber C, Clemente JC, Knights D, Knight R,

447 Gordon JI. 2012. Human gut microbiome viewed across age and geography. Nature

$448 \quad 486: 222-227$.

449 3. Rios-Covian D, Gueimonde M, Duncan SH, Flint HJ, De Los Reyes-Gavilan CG. 2015.

450 Enhanced butyrate formation by cross-feeding between Faecalibacterium prausnitzii and

451 Bifidobacterium adolescentis. FEMS Microbiol Lett 362:1-7.

452 4. Canani RB, Costanzo M Di, Leone L, Pedata M, Meli R, Calignano A. 2011. Potential

453 beneficial effects of butyrate in intestinal and extraintestinal diseases. World J

$454 \quad$ Gastroenterol 17:1519-1528.

455 5. Flint HJ, Duncan SH, Scott KP, Louis P. 2015. Links between diet, gut microbiota 
composition and gut metabolism. Proc Nutr Soc 74:13-22.

457 6. Lopez-Siles M, Khan TM, Duncan SH, Harmsen HJM, Garcia-Gil LJ, Flint HJ. 2012.

458 Cultured representatives of two major phylogroups of human colonic Faecalibacterium

459 prausnitzii can utilize pectin, uronic acids, and host-derived substrates for growth. Appl

$460 \quad$ Environ Microbiol 78:420-428.

461 7. Mitri S, Richard Foster K. 2013. The Genotypic View of Social Interactions in Microbial 462 Communities. Annu Rev Genet 47:247-273.

463 8. Kolter R, Greenberg EP. 2006. Microbial sciences: The superficial life of microbes.

$464 \quad$ Nature 441:300-302.

465 9. Johns NI, Blazejewski T, Gomes ALC, Wang HH. 2016. Principles for designing

466 synthetic microbial communities. Curr Opin Microbiol 31:146-153.

467 10. Turnbaugh PJ, Ley RE, Hamady M, Fraser-Liggett CM, Knight R, Gordon J. 2007. The 468 Human Microbiome Project. Nature 449:804-810.

469 11. Venturelli OS, Carr AC, Fisher G, Hsu RH, Lau R, Bowen BP, Hromada S, Northen T, Arkin AP. 2018. Deciphering microbial interactions in synthetic human gut microbiome communities. Mol Syst Biol 14:e8157.

472 12. Nicholson JK, Holmes E, Kinross J, Burcelin R, Gibson G, Jia W, Pettersson S. 2012.

473 Metabolic Interactions 108:1262-1268.

474 13. Belzer C, Chia LW, Aalvink S, Chamlagain B, Piironen V, Knol J, de Vos WM. 2017.

475 Microbial metabolic networks at the mucus layer lead to diet-independent butyrate and 476 vitamin B12production by intestinal symbionts. MBio 8:1-14.

477 14. Das P, Ji B, Kovatcheva-Datchary P, Bäckhed F, Nielsen J. 2018. In vitro co-cultures of 478 human gut bacterial species as predicted from co-occurrence network analysis. PLoS One 
$13: 1-14$.

480 15. Duncan SH, Barcenilla A, Stewart CS, Pryde SE, Flint HJ. 2002. Acetate utilization and butyryl coenzyme A $(\mathrm{CoA})$ : Acetate-CoA transferase in butyrate-producing bacteria from the human large intestine. Appl Environ Microbiol 68:5186-5190.

16. Louis P, Flint HJ. 2009. Diversity, metabolism and microbial ecology of butyrateproducing bacteria from the human large intestine. FEMS Microbiol Lett 294:1-8. bacterium Faecalibacterium prausnitzii. Gut Microbes 5.

18. Prévoteau A, Geirnaert A, Arends JBA, Lannebère S, Van De Wiele T, Rabaey K. 2015. Hydrodynamic chronoamperometry for probing kinetics of anaerobic microbial

491 19. Sokol H, Pigneur B, Watterlot L, Lakhdari O, Bermudez-Humaran LG, Gratadoux J-J,

496 20. Tremaroli V, Bäckhed F. 2012. Functional interactions between the gut microbiota and host metabolism. Nature 489:242-249.

498 21. Stewart CS, Hold GL, Duncan SH, Flint HJ, Harmsen HJM, Hold GL, Harmsen HJM, Stewart CS, Flint HJ. 2002. Growth requirements and fermentation products of gen. nov., comb. nov. Int J Syst Evol Microbiol 52:2141-2146. 
502 22. Maier, Eva. 2017. Effect of Faecalibacterium prausnitzii on intestinal barrier function and

503 immune homeostasis.

504 23. Vital M, Karch A, Pieper DH. 2017. Colonic Butyrate-Producing Communities in

505 Humans: an Overview Using Omics Data. mSystems 2:e0130-17.

506 24. Wrzosek L, Miquel S, Noordine ML, Bouet S, Chevalier-Curt MJ, Robert V, Philippe C,

507 Bridonneau C, Cherbuy C, Robbe-Masselot C, Langella P, Thomas M. 2013. Bacteroides thetaiotaomicron and Faecalibacterium prausnitzii influence the production of mucus glycans and the development of goblet cells in the colonic epithelium of a gnotobiotic

25. Henson M, Phalak P. 2017. Byproduct Cross Feeding and Community Stability in an In Silico Biofilm Model of the Gut Microbiome. Processes 5:13.

513 26. Heinken A, Sahoo S, Fleming RMT, Thiele I. 2013. Systems-level characterization of a host-microbe metabolic symbiosis in the mammalian gut. Gut Microbes 4.

27. Heinken A, Khan MT, Paglia G, Rodionov DA, Harmsen HJM, Thiele I. 2014. Functional metabolic map of Faecalibacterium prausnitzii, a beneficial human gut microbe. J

28. El-Semman IE, Karlsson FH, Shoaie S, Nookaew I, Soliman TH, Nielsen J. 2014.

521 29. Orth JD, Thiele I, Palsson BØ. 2010. What is flux balance analysis? Nat Biotechnol $28: 245-8$.

523 30. Feist AM, Zielinski DC, Orth JD, Schellenberger J, Markus J, Palsson BØ. 2011. Model- 
coli. Metab Eng 12:173-186.

526 31. Zomorrodi AR, Segrè D. 2016. Synthetic ecology of microbes: mathematical models and applications Graphical Abstract HHS Public Access. J Mol Biol J Mol Biol Febr Pt B $27: 837-861$.

32. Mahadevan R, Edwards JS, Doyle FJ. 2002. Dynamic Flux Balance Analysis of diauxic growth in Escherichia coli. Biophys J 83:1331-1340.

531 33. Harcombe WR, Riehl WJ, Dukovski I, Granger BR, Betts A, Lang AH, Bonilla G, Kar A, Leiby N, Mehta P, Marx CJ, Segrè D. 2014. Metabolic resource allocation in individual microbes determines ecosystem interactions and spatial dynamics. Cell Rep 7:1104-1115.

34. Castro R, Neves AR, Fonseca LL, Pool WA, Kok J, Kuipers OP, Santos H. 2009. Characterization of the individual glucose uptake systems of Lactococcus lactis: Mannose-

537 35. Louca S, Doebeli M. 2015. Calibration and analysis of genome-based models for microbial ecology. Elife 4:1-17.

36. Box GEP. 1950. Problems in the Analysis of Growth and Wear Curves Author ( s ): G . E . P . Box Published by : International Biometric Society Stable URL : http://www.jstor.org/stable/3001781 REFERENCES Linked references are available on

543 37. Segre D, Vitkup D, Church GM. 2002. Analysis of optimality in natural and perturbed

545 38. Mahadevan R, Schilling CH. 2003. The effects of alternate optimal solutions in constraintbased genome-scale metabolic models. Metab Eng 5:264-276.

547 39. Meadows AL, Karnik R, Lam H, Forestell S, Snedecor B. 2010. Application of dynamic 
flux balance analysis to an industrial Escherichia coli fermentation. Metab Eng 12:150160.

40. Jahreis K, Pimentel-Schmitt EF, Brückner R, Titgemeyer F. 2008. Ins and outs of glucose transport systems in eubacteria. FEMS Microbiol Rev 32:891-907.

41. Hewitt CE, Mitchell N, Torgerson DJ, Hewitt C, Torgerson D. 2008. significant Unexpected non-significant results from randomised trials can be difficult to accept . 2325.

42. Snee RD. 1972. American Society for Quality On the Analysis of Response Curve Data On the Analysis of Response Curve Data. Source: Technometrics 14:47-62. of a set of data which cannot be handled by multivariate procedures, namely, the case where $\mathrm{n}$, the number of random vectors, is less t. Phychometrika 24:95-112.

44. Han J, Lin K, Sequeira C, Borchers CH. 2015. An isotope-labeled chemical derivatization method for the quantitation of short-chain fatty acids in human feces by liquid chromatography-tandem mass spectrometry. Anal Chim Acta 854:86-94.

Tables:

\begin{tabular}{|l|l|l|l|}
\hline & Low & Medium & High \\
\hline Coculture & 0.110 & 0.071 & 0.049 \\
\hline B. thetaiotaomicron & 0.034 & 0.065 & 0.112 \\
\hline F. prausnitzii & 0.064 & 0.094 & 0.043 \\
\hline
\end{tabular}


569 Table 1. Table of RMSE between the values in Fig. 1 measured experimentally and predicted by

570 simulations, for monocultures and coculture in three carbon source conditions as described in the

571 text.

\begin{tabular}{lllllllllll}
\hline \multicolumn{3}{c}{ Acetate } & \multicolumn{7}{c}{ Butyrate } \\
\hline Source & Sum Sq. & d.f. & $\begin{array}{l}\text { Mean } \\
\text { Sq. }\end{array}$ & F & Prob>F & Sum Sq. & d.f. & Mean Sq. & F & Prob $>$ F \\
& & & & & & & & & & \\
initial glc/ac & 21.14 & 2 & 10.571 & 0.47 & 0.6277 & 1.3206 & 2 & 0.66032 & 0.69 & 0.5015 \\
mono/co & 115.13 & 1 & 115.131 & 5.1 & 0.0261 & 0.11956 & 1 & 0.11945 & 0.13 & 0.7237 \\
initialglc/ac*mono/co & 76.86 & 2 & 38.43 & 1.7 & 0.1877 & 0.275 & 2 & 0.13751 & 0.14 & 0.8655 \\
Error & 2304.56 & 102 & 22.594 & & & 96.9437 & 102 & 0.95043 & & \\
Total & 2517.69 & 107 & & & & 98.6588 & 107 & & \\
\hline
\end{tabular}

Table 2. Analysis of Variance of Shape of Metabolite Curves.

\begin{tabular}{lllllllllll}
\hline & Acetate & \multicolumn{7}{c}{ Butyrate } \\
Source & Sum Sq. & d.f. & $\begin{array}{l}\text { Mean } \\
\text { Sq. }\end{array}$ & F & Prob>F & Sum Sq. & d.f & Mean Sq. & F & Prob> \\
& & & & & & & F & & \\
initial glc/ac & 20.8263 & 1 & 20.8263 & 17.66 & 0.0012 & 0.17635 & 1 & 0.17635 & 4.29 & 0.0606 \\
mono/co & 1.6629 & 2 & 0.8315 & 0.7 & 0.5135 & 2.09982 & 2 & 1.04991 & 25.53 & 0 \\
initialglc/ac*mono/co & 13.2214 & 2 & 6.6107 & 5.61 & 0.0191 & 0.41137 & 2 & 0.20568 & 5 & 0.0263 \\
Error & 14.1527 & 12 & 1.1794 & & & 0.49346 & 12 & 0.04112 & \\
Total & 49.8634 & 17 & & & & 3.181 & 17 & & \\
\hline
\end{tabular}

Table 3. Analysis of Variance of Average Rate of Metabolite Curves.

\begin{tabular}{lllllllllll}
\hline & Acetate & \multicolumn{7}{c}{ Butyrate } \\
\hline Source & Sum Sq. & d.f. & Mean Sq. & F & Prob $>$ F & Sum Sq. & d.f. & $\begin{array}{l}\text { Mean } \\
\text { Sq. }\end{array}$ & F & Prob $>$ F \\
& & & & & & & & & & \\
initial glc/ac & $1.81 \mathrm{E}+07$ & 2 & $9.06 \mathrm{E}+06$ & 0.08 & 0.9272 & 2745098 & 2 & 1372549 & 0.46 & 0.6328 \\
mono/co & $4.80 \mathrm{E}+06$ & 1 & $4.80 \mathrm{E}+06$ & 0.04 & 0.8417 & 25019.6 & 1 & 25019.6 & 0.01 & 0.9272 \\
initialglc/ac*mono/co & $9.61 \mathrm{E}+06$ & 2 & $4.81 \mathrm{E}+06$ & 0.04 & 0.9606 & 953798 & 2 & 476899 & 0.16 & 0.8526 \\
Error & $1.22 \mathrm{E}+10$ & 102 & $1.20 \mathrm{E}+08$ & & & $3 \mathrm{E}+08$ & 102 & 2985539 & \\
Total & $1.23 \mathrm{E}+10$ & 107 & & & & $3.1 \mathrm{E}+08$ & 102 & & \\
\hline
\end{tabular}

Table 4. Analysis of Variance of Average Rate of Metabolite Curves. 


\begin{tabular}{lllllllllll} 
Source & Sum Sq. & d.f & Mean Sq. & F & Prob> & Sum Sq. & d.f. & $\begin{array}{l}\text { Mean } \\
\text { Sq. }\end{array}$ & F & Prob>F \\
initial glc/ac & 162363.1 & 1 & 162363.1 & 1.57 & 0.234 & 11506.9 & 1 & 11506.9 & 0.2 & 0.6612 \\
mono/co & 3919429.3 & 2 & 1959714.6 & 18.96 & 0.0002 & 1156514 & 2 & 578257 & 10.15 & 0.0026 \\
initialglc/ac*mono/co & 2590821 & 2 & 1295410.5 & 12.53 & 0.0012 & 517152 & 2 & 258576 & 5.54 & 0.0341 \\
Error & 12640529.9 & 12 & 103377.5 & & & 683892 & 12 & 56991 & & \\
Total & 7913143.3 & 17 & & & & 2369065 & 17 & & \\
\hline
\end{tabular}

Table 5. Analysis of Variance of Average Rate of Biomass-Normalized Metabolite Curves.

\begin{tabular}{|c|c|c|c|c|}
\hline Species & $V \max [\mathrm{mmol} / \mathrm{hg}]$ & Glc./Ac.Vmax $[\mathrm{mmol} / \mathrm{hg}]$ & $\begin{array}{l}\text { Km } \\
{[\mathrm{mM}]}\end{array}$ & $\begin{array}{l}\text { Glc./Ac. Km } \\
{[\mathrm{mM}]}\end{array}$ \\
\hline B.thetaiotaomicoron & 11 & 11 & 3 & 3 \\
\hline F.prausnitzii & 23 & 10 & 10 & 5 \\
\hline
\end{tabular}

589 Table 6. Parameters of the Michaelis-Menten uptake functions. Glucose and acetate uptake

590 parameters were obtained independently from the rest of the nutrients for the model of $F$.

591 prausnitzii.

\section{Supplemental table legend:}

594 Table S1. AB Sciex 4000-TRAP parameters used for detection of SCFA Q1 - m/z of the analyte

595 ion detected on the 1rst quadrupole.Q3 - m/z of the anatyte's fragment ion detected on the third

596 quadrupole. DP- declustering potential, CE - collision energy. Analytes in red are internal

597 standards.

Figure legends:

Figure 1. Optical densities for single species and coculture of $F$. prausnitzii and $B$.

601 thetaiotaomicron, grown in three different media conditions. The simulations (solid curves) were 
602

603

604

605

606

607

608

609

610

611

612

613

614

615

Figure 3. Experimental and simulated (solid, dot and dash curves) butyrate production

time courses for monocultures and coculture under the three initial glucose/acetate initial

concentrations. Apparent differences demonstrated in these plots in butyrate production for F.

prausnitzii monoculture vs. coculture and for the three initial concentrations can be tested

statistically using ANOVA on summary statistics describing the curves. The simulated curves

correspond to the maximized (solid curve) and minimized (dash dot curve) butyrate secretion.

624 butyrate secretion. 
627 monocultures and coculture under the three initial glucose/acetate initial concentrations.

628 Apparent differences demonstrated in these plots in butyrate production for $F$. prausnitzii

629 monoculture vs. coculture and for the three initial concentrations can be tested statistically using

630 ANOVA on summary statistics describing the curves.

631

Figure 6. A) Butyrate concentration time profile, normalized by the simulated $F$.

prausnitzii biomass, for coculture and monoculture. B) Simulated species composition.

635 Supplemental figure legends:

Figure S1. Simulated glucose concentration.

Figure S2. Biomass normalized acetate concentration.

Figure S3. Simulated fluxes of key reactions in the butyrate fermentation pathway in $F$. prausnitzii, for high glucose concentration, at time zero and 5 hours.

Figure S4. Simulated butyrate secretion fluxes in $F$. prausnitzii, under secondary

641 maximization/minimization of butyrate or lactate production, as a function of phosphate

642 concentration, in low glucose conditions. 


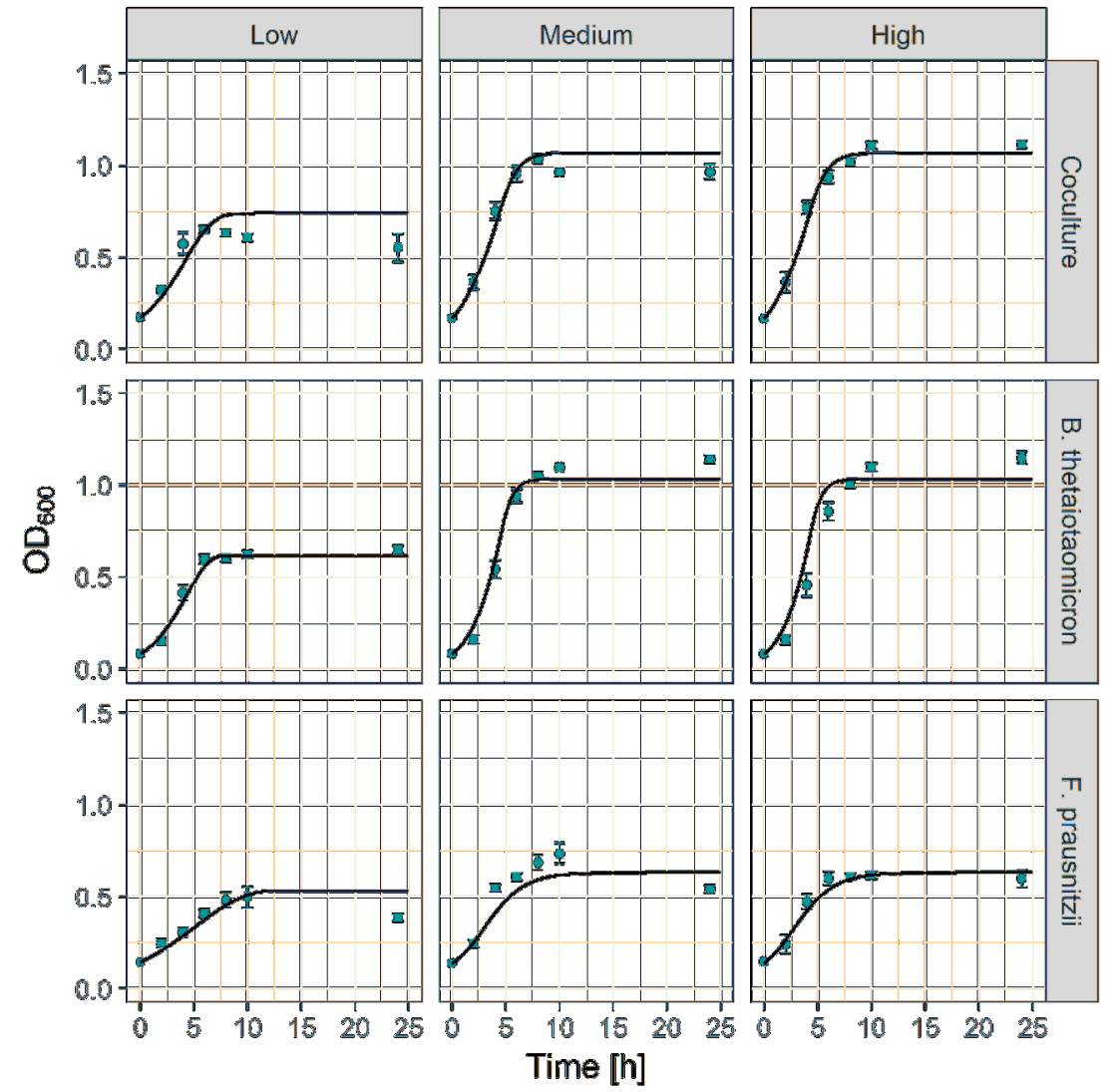

Figure 1. Optical densities for single species and coculture of $F$. prausnitzii and $B$. thetaiotaomicron, grown in three different media conditions. The simulations (solid curves) were obtained by dFBA with the same set of uptake parameters for all media conditions. The columns correspond to the media conditions while the rows correspond to the cultured species. 
A

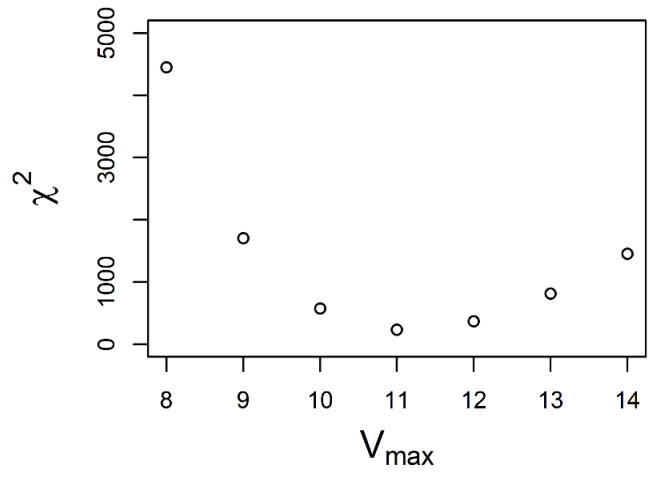

B

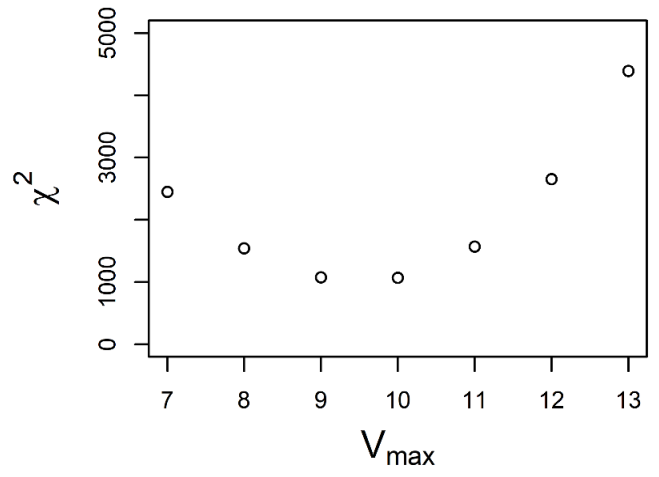

C

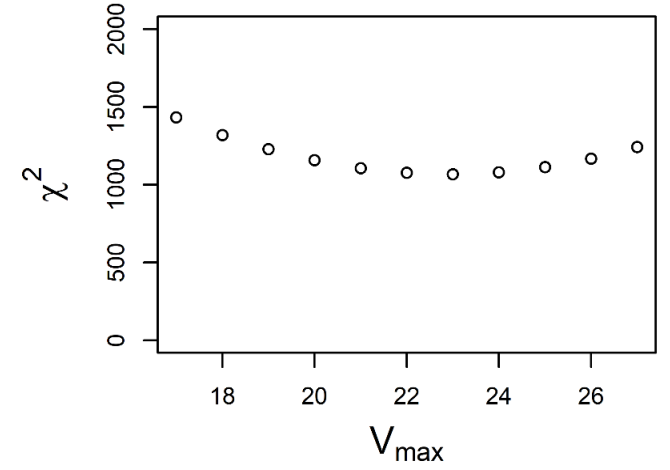

Figure 2. The sensitivity of the OD curves to the values of the maximum nutrient uptake parameter $V_{\max }$. The values of the $V_{\max }$ parameter used in the simulations were obtained by minimizing $\mathrm{c}^{2}$,the sum of the squared deviations of the simulation from the experimental values, weighted by the

measured variance. We used a single value of $V_{\max }$ for the uptake of all nutrients by the $B$. thetaiotaomicron model, with the minimum of minimizing $\mathrm{c}^{2}$, shown in panel A). In the case of $F$. prausnitzii, we determined two separate values of minimizing $\mathrm{c}^{2}$, one for glucose and acetate uptake shown on panel B), and another one for the rest of the nutrients, shown on panel C). 


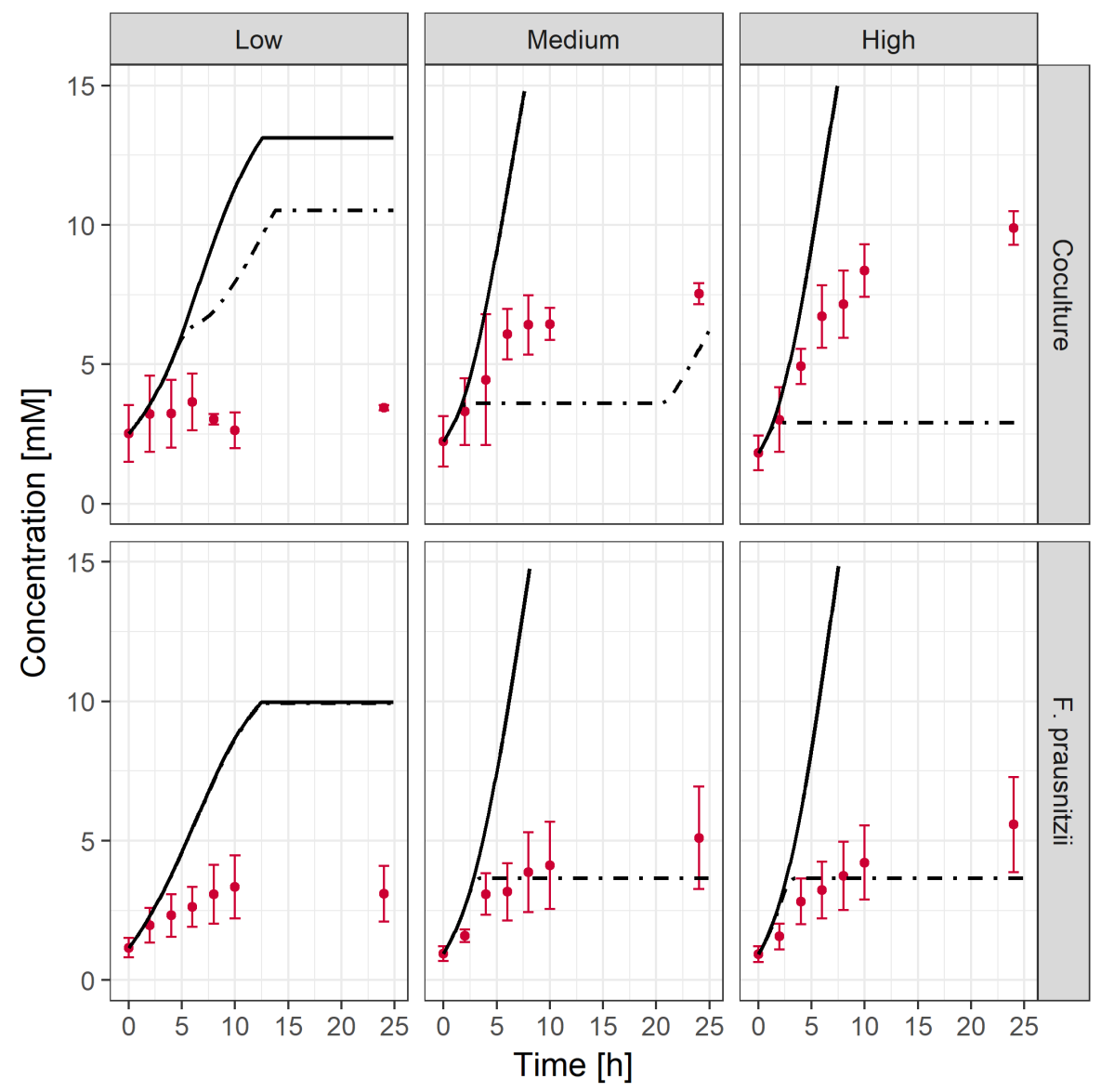

Figure 3. Experimental and simulated (solid, dot and dash curves) butyrate production time courses for monocultures and coculture under the three initial glucose/acetate initial concentrations. Apparent differences demonstrated in these plots in butyrate production for $F$. prausnitzii monoculture vs. coculture and for the three initial concentrations can be tested statistically using ANOVA on summary statistics describing the curves. The simulated curves correspond to the maximized (solid curve) and minimized (dash dot curve) butyrate secretion. 


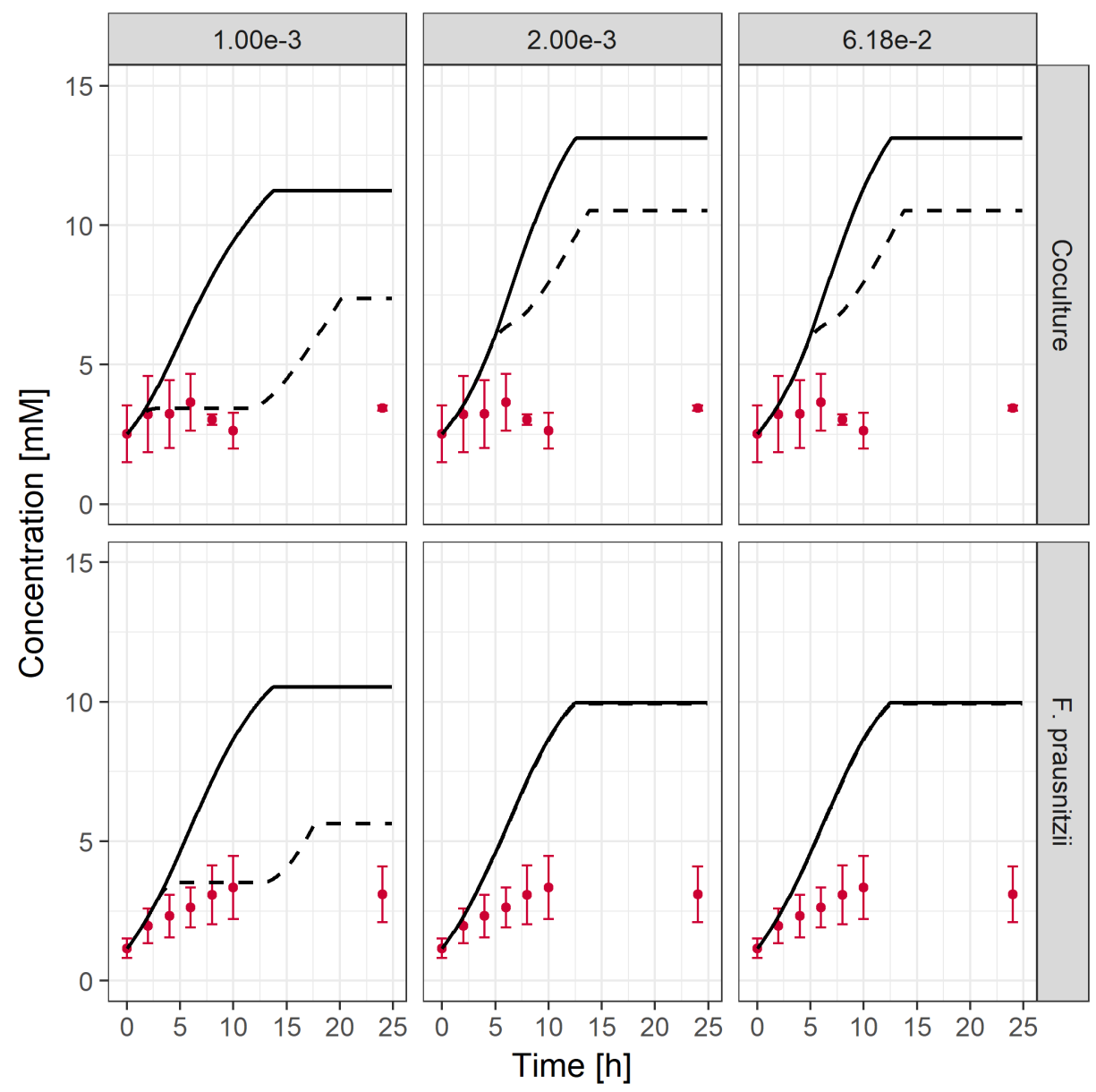

Figure 4. Simulated butyrate production for three starting abundances of phosphate for the low initial acetate/glucose concentration. Lowering of the phosphate concentration leads to multiple FBA solutions and difference between secondary minimization and maximization of butyrate secretion. 


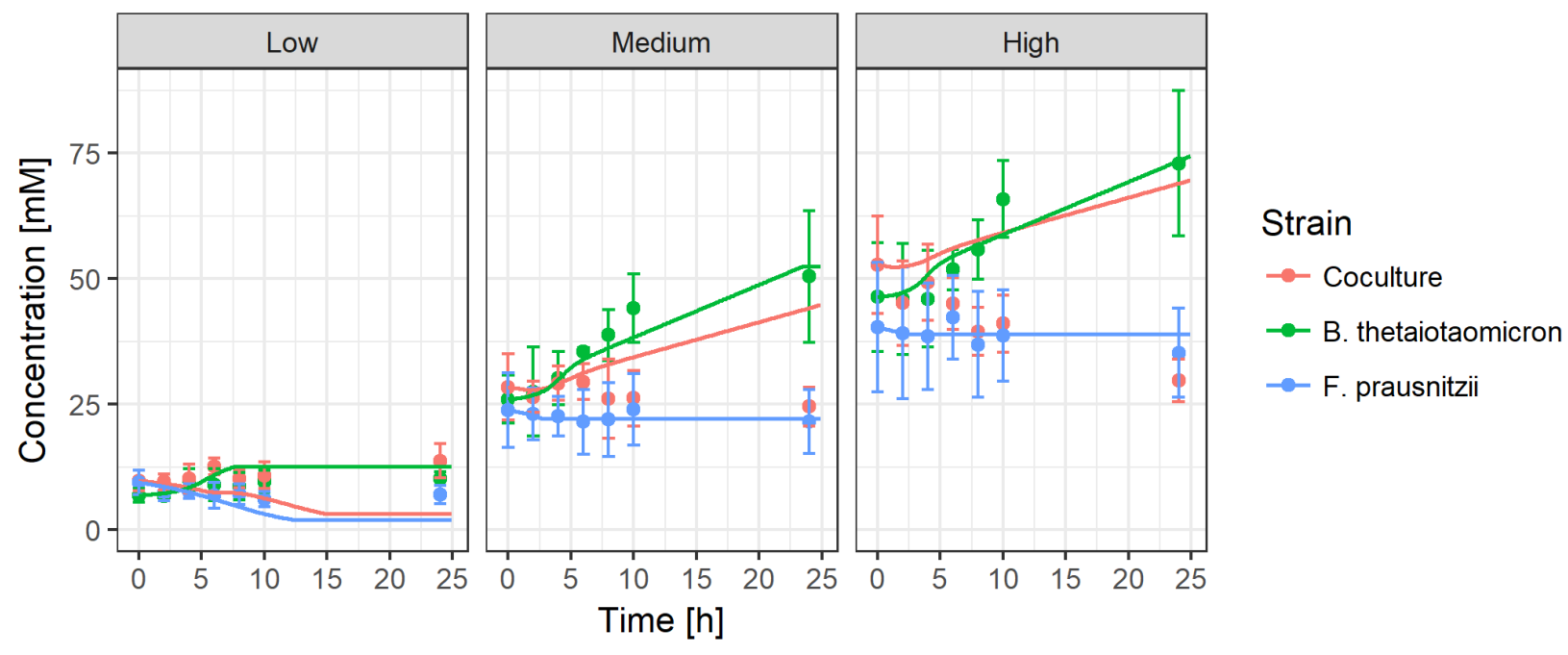

Figure 5. Experimental and simulated (solid curves) acetate production time courses for monocultures and coculture under the three initial glucose/acetate initial concentrations. Apparent differences demonstrated in these plots in butyrate production for $F$. prausnitzii monoculture vs. coculture and for the three initial concentrations can be tested statistically using ANOVA on summary statistics describing the curves. 
A
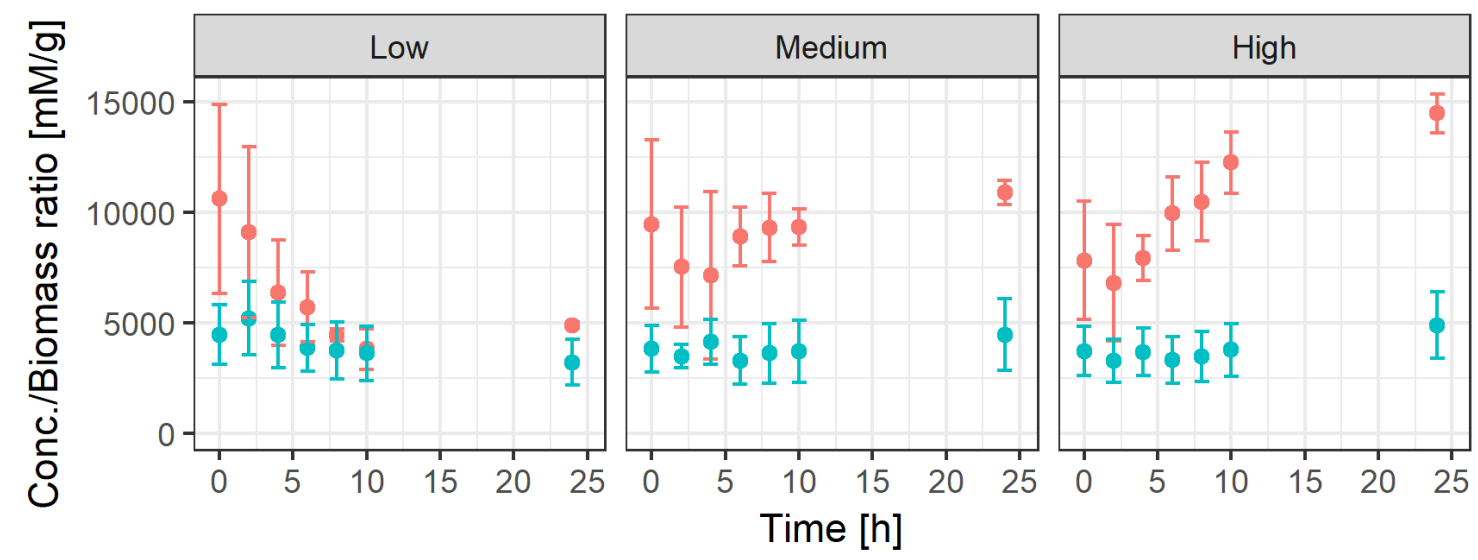

\section{Species}

$\rightarrow$ Coculture

$\rightarrow$ F. prausnitzii

B
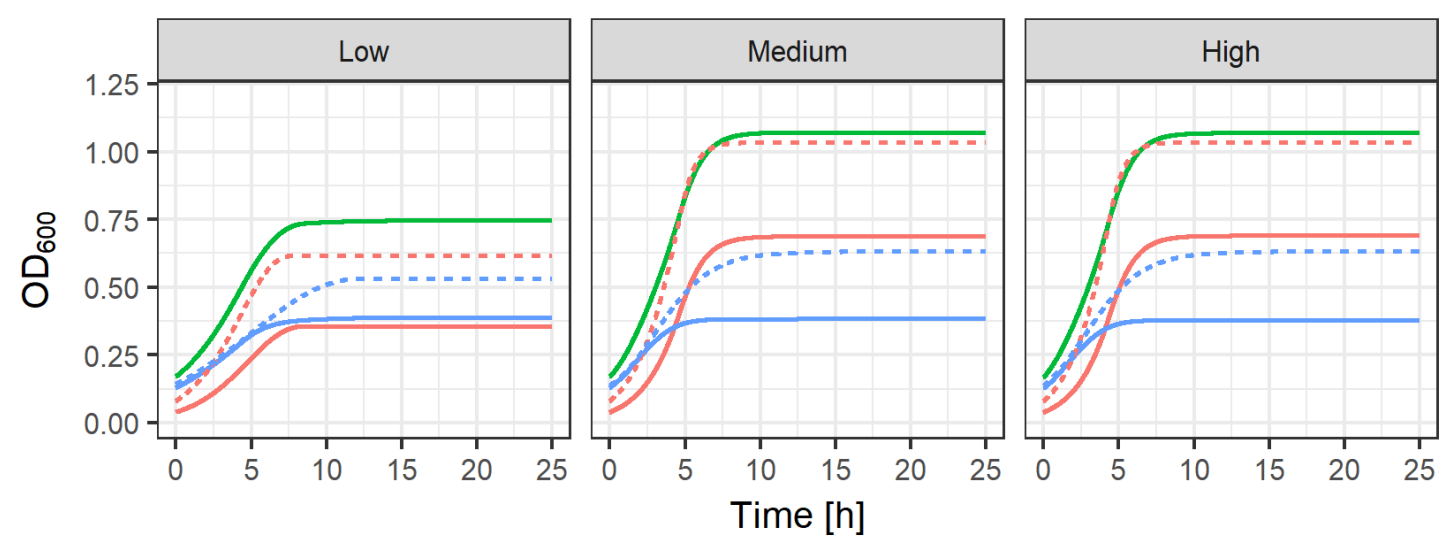

Species

- B. thetaiotaomicron

- Coculture

— F. prausnitzii

linetype

- Coculture

-.- Monoculture

Figure 6. A) Butyrate concentration time profile, normalized by the simulated $F$. prausnitzii biomass, for coculture and monoculture. B) Simulated species composition. 